\title{
Comparison between 2D and 3D codes in dynamical simulations of gas flow in barred galaxies
}

\author{
I. Pérez $z^{1 \star, 2 \star \star}$ \\ ${ }^{1}$ Kapteyn Astronomical Institute, University of Groningen, The Netherlands \\ e-mail: isa@astro.rug.nl \\ 2 Departamento de Física teórica y del Cosmos, Universidad de Granada, Spain
}

Received 28 June 2007 / Accepted 11 October 2007

ABSTRACT

\begin{abstract}
Context. One of the ways to determine the contribution of the dark halo to the gravitational potential of a galaxy is study non-circular (streaming) motions and the associated gas shocks in the bar region. These motions, determined by the potential in the inner parts, can break the disk-halo degeneracy. Here, two main fluid dynamical approaches have been chosen to model the non-circular motions in the bar region; a 2D Eulerian grid code for an isothermal gas (FS2) and a 3D smoothed particle hydrodynamic code $(N$-body/SPH) Aims. The aim of this paper is to compare and quantify the differences in the gas flows of rotating barred potentials obtained using two different fluid dynamical approaches.

Methods. We analysed the effect of using 2D and a 3D codes in calculating the gas flow in barred galaxies and to what extent the results are affected by the code. To do this, we derived the velocity field and density maps for the mass model of NGC 4123 using a 3D $N$-body/SPH code and compare the results to the previous 2D Eulerian grid code results. Numerical modelling, 3D $N$-body/SPH simulations

Results. The global velocity field and the gas distribution are very similar in both models. The study shows that the position and strength of the shocks developed in the SPH simulations do not vary significantly compared to the results derived from the 2D FS2 code. The largest velocity difference across the shock is $20 \mathrm{~km} \mathrm{~s}^{-1}$ between the $2 \mathrm{D}$ and $3 \mathrm{D}$ fluid dynamical models.

Conclusions. The results when deriving the dark matter content of barred galaxies using the bar-streaming motions and strength and position of shocks are robust to the fluid dynamical model used. The effect of 2D and 3D modelling can be neglected in this type of study.
\end{abstract}

Key words. methods: numerical - Galaxy: kinematics and dynamics - cosmology: dark matter

\section{Introduction}

There have been several studies addressing the distribution of dark matter in galaxies using the non-axisymmetry of the potential of barred galaxies: a) the fluid dynamics approach with the work by Weiner et al. (2001a, hereafter WSW01) for modelling NGC 4123; Pérez (2003) and Pérez et al. (2004, hereafter PFF04) for the study of the dark matter content of 5 barred galaxies (NGC 5505, NGC 7483, NGC 5728, and NGC 7267); and b) the sticky-particle approach by Rautiainen et al. (2004) modelling the dynamics of ESO 566-24, and Salo et al. (1999), who modelled the $\mathrm{H} \alpha$ velocity field of IC 4214 to derive the halo contribution.

The streaming motions and the associated gas shocks in the bar region are determined by the potential in the inner parts of galaxies. The velocity field of an axisymmetric galaxy does not allow the contribution of the halo to be uniquely disentangle from that of the disk. The signatures of non-circular motions, however, can break the disk-halo degeneracy and be used to obtain the contribution of the dark halo to the potential. Common to all the modelling, the stellar potential is generated directly from the broad-band galaxy images with some recipe for treating the vertical distribution and some assumptions about the mass-tolight ratio $(M / L)$ of the stellar populations. The velocity fields

\footnotetext{
$\star$ Veni Fellow.

$\star \star$ Associate Researcher.
}

obtained in this way are then compared to the observed kinematic information to determine if they can be reproduced by the models.

The main result, common to all the modelling carried out up to now, is that the gravitational field in the inner region is mostly provided by the stellar luminous component. The bar pattern speeds found by the different groups are consistent with fast rotators, and the best-fitting $M / L$ are compatible with $M / L$ derived from current population synthesis models. Although it is a very powerful method, only a handful of galaxies have been modelled in this way.

Two main fluid dynamical approaches have been chosen for this type of modelling: a Eulerian grid code for an isothermal gas (FS2) and a smoothed particle hydrodynamic code ( $N$-body/SPH). The SPH is a Lagrangian method for solving Euler's equation of motion. In this technique, the system is represented by a set of particles, and the gas properties are calculated by a weighted average over the neighbouring particles. On the other hand, the Eulerian grid is fixed in space and time, where the grid nodes and cells remain spatially fixed while the materials flow through the mesh. In the modelling carried out by WSW01, the scale height is introduced as a smoothing length for the potential, while the approach chosen by PFF04 uses a full 3D calculation of the forces. For future work and as a consistency check, it is important to understand whether the results obtained are a) dependent on the dynamical code used and b) 2D versus 
3D. No significant differences are expected between the SPH and the Eulerian grid approaches. Previous simulations (Englmaier \& Gerhard 1997; Patsis \& Athanassoula 2000, hereafter PA00), running 2D SPH in the same conditions as used by Athanassoula (1992) with the FS2 Eulerian grid code indicated that the main features, such as the strength of the shocks in the bar, are quite similar and small differences arise due to the statistical nature of SPH or to the numerical and artificial viscosities used in the different codes. Both studies point out the importance of certain parameters used in the simulations for the outcome of the modelling, such as the sound speed and the way the nonaxisymmetric part of the potential is introduced. The use of a $2 \mathrm{D}$ versus a $3 \mathrm{D}$ code might introduce large variations in the radial forces due to the different treatments of the vertical forces, which in turn might vary the outcome of the analysis and the conclusions derived from this methodology. In this paper, we investigate the effect of $2 \mathrm{D}$ vs. $3 \mathrm{D}$ numerical modelling in the gas flows of rigidly rotating bar potentials. In order to test this effect, we have modelled the gas flow in NGC 4123 using the mass model and images provided by B. J. Weiner, but also the 3D $N$-body/SPH code used in PFF04. The parameters chosen for the SPH simulations were taken from WSW01. In future work, we will address the comparison between the sticky particle codes and the fluid dynamical methods. The modelling is presented in Sect. 2, and the results and comparison are presented in Sect. 3. Finally, discussion and conclusions are presented in Sect. 4

\section{Modelling}

The methodology for this study is simple. We derive the mass model for NGC 4123 in a way similar to that of WSW01 and then run the SPH models to obtain the gas distribution and velocity field, using the best-fit parameters $(M / L$ and bar pattern speed) in WSW01. And finally, the gas distribution and the velocity field are compared to the results presented in WSW01. In this section we present a brief summary of both codes and initial conditions, emphasising the SPH carried out in this work and explaining the way in which we derive the mass distribution.

The FS2 code is a second-order, flux-splitting, Eulerian grid code for an isothermal gas in an imposed gravitational potential, originally written by van Albada (1985). The sound speed of the gas is $8 \mathrm{~km} \mathrm{~s}^{-1}$. The code does not include the self-gravity of the gas. The barred potential rotates at a fixed pattern speed $\Omega_{\mathrm{B}}$, and the grid rotates with the bar. In WSW01, 0.1 Gyr are allowed to fully grow the bar in order to steadily adjust the flow. The initial gas surface density is set to be $10 M_{\odot} \mathrm{pc}^{-2}$ inside a radius of $8 \mathrm{kpcs}$, and it falls off exponentially outside that radius. A $\operatorname{sech}^{2}\left(z / z_{0}\right)$, where $z_{0}$ is the scale height, is assumed for the vertical distribution. With this distribution, the accelerations are calculated in the midplane using a Fourier transform method to convolve a Green's function with the surface brightness distribution (Hockney 1965). The scale height is effectively a smoothing length for the potential. For a complete explanation of the code and the initial conditions, refer to WSW01.

The 3D $N$-body and hydro code was initially developed by the Geneva Observatory galactic dynamics group for spiral galaxy studies (Pfenniger \& Friedli 1993; Fux 1997, 1999). The initially self-consistent code was modified to use a fixed rotating potential. The stellar potential is fixed using the observed light distribution. The potential is calculated using a particlemesh technique with cylindrical-polar grid and the short-range forces are softened using a variable homogeneous ellipsoid kernel with principal axes matched to the local grid resolution. The pressure forces and viscous forces are derived by 3D smooth particle hydrodynamics, SPH (Benz 1990; Fux 1999). The gas is taken to be isothermal with a sound speed of $10 \mathrm{~km} \mathrm{~s}^{-1}$ and an adiabatic index of $5 / 3$, corresponding to the atomic hydrogen. For more details about the code, refer to Fux (1999) or to his Ph.D. Thesis, Fux (1997). For details about the code in the form used for this work, refer to PFF04.

The grid size used is 95 cells in the radial, 96 in the azimuthal, and 1214 (including doubling up) in the vertical directions. The vertical resolution is set to 0.05 times the scale height adopted for the luminous mass distribution. The number of gas particles used is 300000 . The barred potential rotates at fixed pattern speed $\Omega_{\mathrm{B}}$ and there is no self-gravity. These simulations were run; first, using the Alpha Server Sc system at the Australian National University super computer facility, and later the Cray SV1e super computer at Groningen University.

For the initial conditions, see PFF04. The initial gas density in the simulations consists of one component. The radial distribution for the gas is a Beta function with a standard deviation set to the scale-length of the visible disk and radially vanishing at a distance 4 times this scale-length. The scale length assumed for NGC 4123 is $3.2 \mathrm{kpc}$, as derived from the $I$-band luminosity distribution in Weiner et al. (2001b). The vertical distribution of the gas is generated directly by solving the hydrostatic equilibrium equation for an isothermal gas. The gas particles have pure circular motion with cylindrical rotation and zero velocity dispersion, since the effective dispersion is taken into account in the pressure component of the SPH. The bar is chosen to grow linearly during three bar rotations, so that the gas flow can steadily adjust to the forcing bar without requiring much $\mathrm{CPU}$ time. In previous simulations (PFF04), different onset times were checked to ensure that the particle flow adjusted steadily at the chosen onset time, finally setting the onset time to 3 bar rotations.

We used the $I$-band light distribution, and exponential vertical profile with a scale height of $200 \mathrm{pc}$ to derive the potential in a similar fashion to WSW01. They constructed a bi-symmetric image by rotating the image $180^{\circ}$ and averaging the rotated and original images. The central point source was removed and modelled as a spherical Gaussian distribution $\rho(r) \propto \exp \left(-r^{2} / 2 r_{\mathrm{c}}^{2}\right)$ with a scale radius $\left(r_{\mathrm{c}}\right)$ of $200 \mathrm{pc}$; refer to WSW01 for details about the mass distribution. Since the model used for comparison is the maximum disk model, no dark matter halo profile was included in the derivation of the mass. Because the potential in the bar region is dominated by the stellar component, we do not expect a significant difference in the results when excluding this component. The model parameters used were $M / L$ of 2.25 and a pattern speed of $20 \mathrm{~km} \mathrm{~s}^{-1} \mathrm{kpc}^{-1}$, corresponding to a $R_{\text {cor }} / R_{\text {bar }}=1.35$, where $R_{\text {cor }}$ is the corotation radius and $R_{\text {bar }}$ the bar semi-major axis. These parameters correspond to the bestfit model in WSW01. To derive the velocity field, we used the parameters derived by Weiner et al. (2001b) from their kinematic data; i.e. $i=45^{\circ}$ and major axis of the galaxy at $57^{\circ}$ north through west. Both codes were run without gas self-gravity. Low-resolution simulations have shown that gas self gravity is irrelevant for the results in this work (Pérez 2003; Pérez et al. 2004).

The gas density distribution for the SPH modelling clearly settles into a stationary configuration after 3 bar rotations, and a nuclear ring develops perpendicular to the bar, associated to the $x_{2}$ family of orbits. From the frequency plot of the axisymmetric case, we see that NGC 4123 possesses two ILR for the derived pattern speed. The presence of the ILR has already been suggested in Weiner et al. (2001b) from the offset of the dust lanes in the colour images of NGC 4123. 

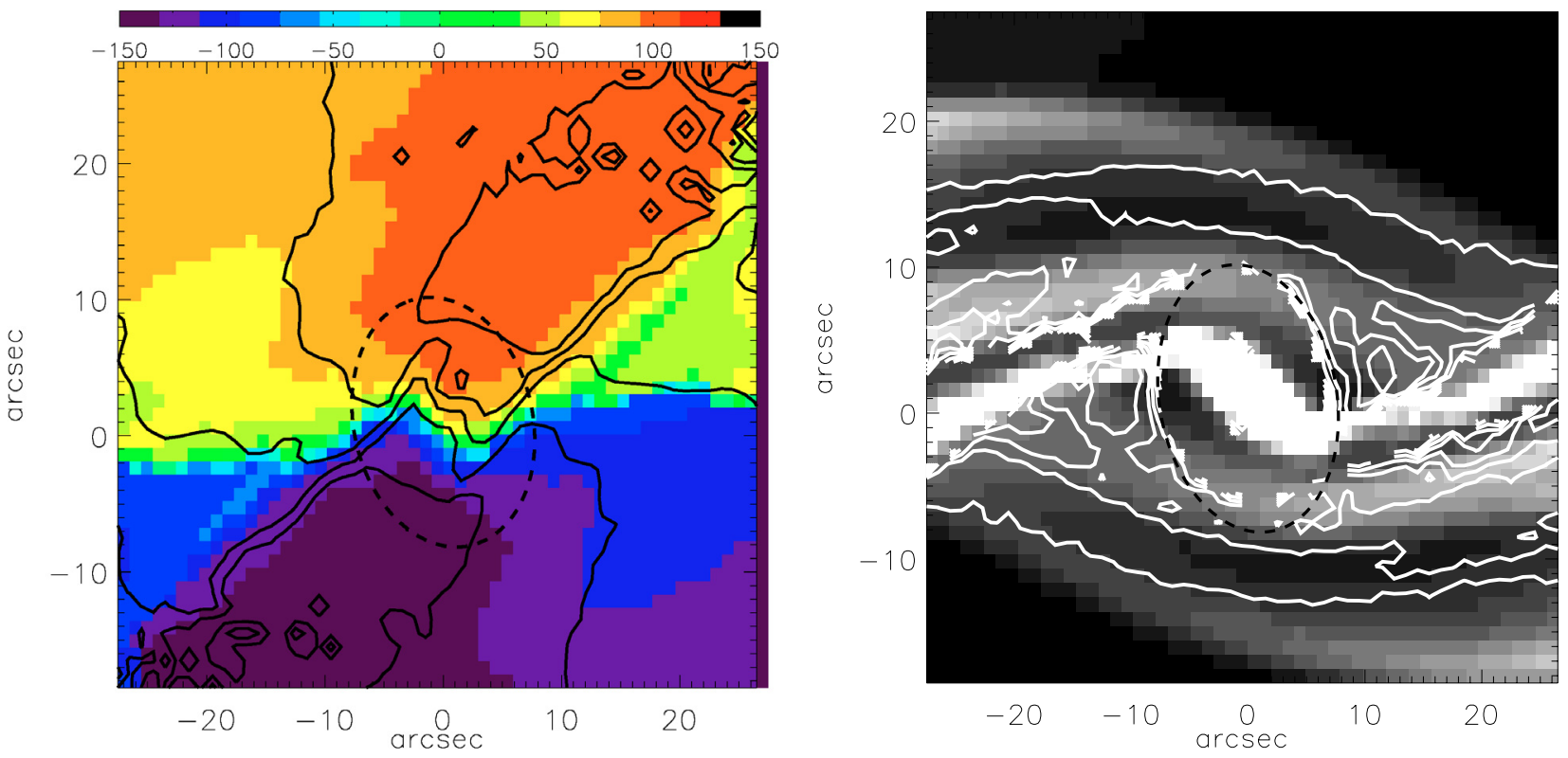

Fig. 1. In the left panel a) the velocity field contours in the bar region, projected onto the plane of the sky, using $i=45^{\circ}$ and major axis of the galaxy at $57^{\circ}$ northwest (values from WSW01). Superposed are the isovelocity contours of the projected velocity field as derived in this work, with levels corresponding to intervals of $30 \mathrm{~km} \mathrm{~s}^{-1}$. In the right panel b) density distribution from WSW01, superposed are the isodensity contours from the particle distribution of the SPH for the mass distribution of NGC 4123 derived from the $I$-band after 4 bar rotations. The non-axisymmteric component is fully grown after three bar rotations and the bar pattern speed is rotating clockwise in the inertial frame. Notice the agreement in the regions of high density within the bar region.

\section{Comparison and results}

After three bar rotations, when the non-axisymmetric component is fully grown, the gas distribution settles into a steady configuration until the end of the simulation, six bar rotations. The gas distribution at the end of the simulation is shown in Fig. 1. The region chosen for a comparison between the two models is the region used by WSW01 in their study (see Fig. 1 for the location of the comparison regions). They chose the bar region because it is the area where the non-axisymmetric motions are strong and excluded the central region because the velocity gradients are very high and probably unresolved. Their dynamical resolution is worse at the centre because there are few cells across the scale of a streamline. Therefore, for a meaningful comparison between the models, we restrict the comparison to the regions discussed in WSW01.

Previous work (PA00), in a study of the effect of hydrodynamical and numerical parameters in the simulations of gas flow in barred galaxies, noticed that differences in the sound speed would change the gas distribution morphology and the inflow rate of the gas. The two models under comparison here have similar sound speeds $\left(10 \mathrm{~km} \mathrm{~s}^{-1}\right.$ for the SPH models and $8 \mathrm{~km} \mathrm{~s}^{-1}$ for the grid based models); therefore, we do not expect differences arising from this effect.

Another parameter that could cause a difference between the two models is the way the non-axisymmetric component is introduced. PA00 noticed that introducing the bar abruptly results in a different morphology when compared to the case where the bar is slowly introduced. In both models analysed in here, the bar is introduced slowly, allowing the flow to steadily adjust to the growth of the bar.

The spatial gas resolution in the two codes is very different and difficult to compare. The gas resolution in the SPH is determined by the particle smoothing length, in this case, to ensure good resolution in high-density regions, the smoothing length is assigned to each particle such that their number of neighbouring particles always remains close to a fixed number. The average gas smoothing length is close to the vertical gravitational resolution, which is $20 \mathrm{pc}$ for NGC 4123 . However, in the central parts, where the particle density is high, the resolution reaches a few parsecs. The resolution of the grid based code is $84.7 \mathrm{pc}^{2}$, using a grid having $256 \times 512$ cells. It is interesting to notice that, while the gas distribution in the SPH code develops a central ring, associated to the ILR, the gas density distribution does not present this central feature in the results obtained with the Eulerian code; although, the underlying potential and the sound speed are similar in both models. Because the mass distribution and the bar pattern speed are similar, the difference in whether the central ring is present is possible due to differences between the two codes. PA00 showed the gas distribution for a series of 2D SPH models with different gas sound speeds and compared them to simulations carried out with a 2D second-order flux-splitting code similar to the one used by WSW01. The gas response for a sound speed of $10 \mathrm{~km} \mathrm{~s}^{-1}$ was similar in both cases, both cases developing a nuclear ring. However, for a sound speed of $15 \mathrm{~km} \mathrm{~s}^{-1}$, the gas density response is different in both codes. The gas density in the SPH code develops a central $x_{2}$ ring, while the gas distribution in the eulerian code gives no central ring. This should be further investigated using 2D (or 3D) consistently. The comparison we have carried out throughout this paper is made outside this central region. Future high-resolution observations of the gas distribution in the central parts would show whether the velocity fields and the density distribution generated in our modelling is a good representation of the central region in NGC 4123, whether we are making wrong assumptions on the vertical thickness of the bar or whether the dynamical approach used here is not appropriate to the problem of modelling the central regions.

The general characteristics of the velocity fields are the following; the offset shocks in the bar are roughly east-west and nearly perpendicular to the bar where there is a large velocity gradient. In Fig. 1b one can see that, within the comparison 

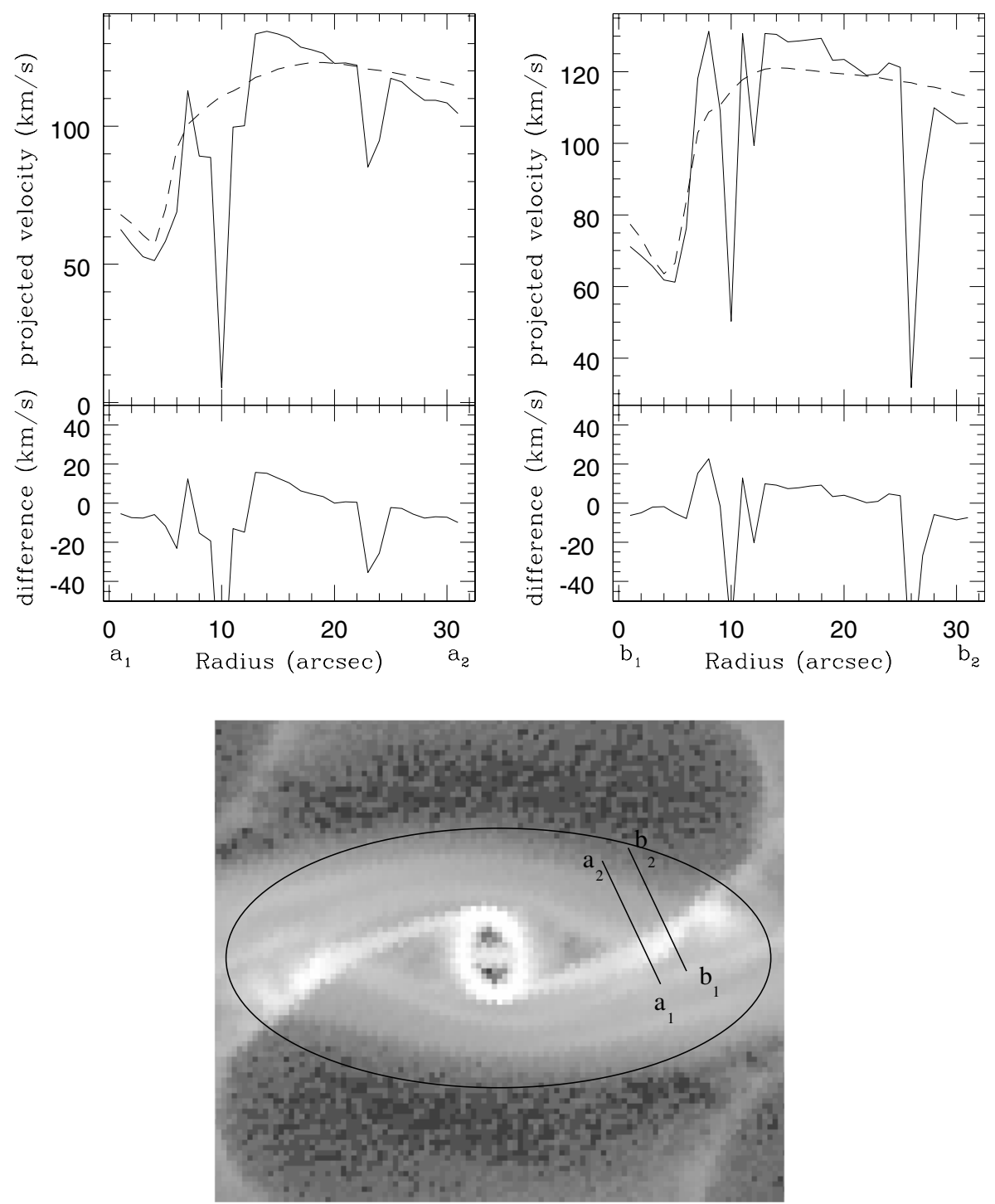

Fig. 2. Two cuts across the high velocity gradient region for the deprojected velocity maps of both models. The top panels show the projected velocity. The position corresponds to the velocity going from the leading side towards the trailing side of the shock region in a 30 pixel range $(\approx 23.4$ arcsec $)$, with a cut at a distance along the shock of 30 pixels from the centre (left panel) and a distance of 35 pixels from the centre (right panel). The middle panels correspond to the residuals of both line-of-sight velocity distributions. The bottom panels shows the position of the cuts (two parallel black lines) shown in the above panels perpendicular to the shock region on top of the general gas distribution.

region, i.e. the regions associated to the dust lanes, the agreement between both models is very good. These regions are associated to shocks and to regions of high velocity gradients.

The velocity field and the corresponding velocity gradients are similar in both models as shown in Fig. 1a. The shape of the density maxima (accompanied by the high velocity gradients) puts strong constraints on the potential (Athanassoula 1992). The angle between the high velocity gradient region and the bar is the same in both simulations and the shape of the shock regions in both models is similar, supporting the results from both models. There seems to be a slight displacement of the peak position of the large gradient region between both models $(\approx 1.5 \mathrm{arcsec})$, and in the SPH the regions of highest density are broader than in the FS2 code possibly due to the shock being slightly less well resolved in the SPH model. This difference between SPH and the grid codes has been already noticed Englmaier \& Gerhard (1997). They obtained broader density peaks in the shock regions in 2D SPH modelling compared to $2 \mathrm{D}$ grid models.

To compare the two models more quantitatively, we made cross section profiles of this region of high velocity gradients (see Fig. 2). To facilitate the comparison of the velocity jumps in the shock region, the velocity distribution has been shifted by two pixels (1.56 arcsec) because this is the offset found between both models in the peak position of the gradient. The cut positions have been chosen to be within the comparison region. One has to keep the statistical nature of SPH in mind when comparing the cuts. This causes some particles to have very different values from the general distribution. The velocity jumps are very similar for both models with a maximum velocity difference between the two models across this region of $20 \mathrm{~km} \mathrm{~s}^{-1}$. The amplitude of the velocity jump depends on the mass distribution in the bar region, with thinner and more massive bars having larger velocity jumps (Athanassoula 1992) and, of course, in this case, also on the position of the cut across the shock. This is what gives rise to the difference between the right and the left panels in Fig. 2. Overall, the shape and the amplitude of the gas velocity crosssection profiles between the two models agree very well.

A change in the parameters, such as the pattern speed and the $M / L$, would have caused the velocity field and the shock strength, location and shape to be significantly different (Athanassoula 1992; Weiner et al. 2001a; Pérez et al. 2004) to the results obtained here.

There is a discrepancy outside the bar region, and the position and shape of the spiral arms do not agree between the two models. In the Eulerian code the arms are narrower than in the 
SPH code and seem to circumscribe the arms formed in the SPH code. In any case, the region of the spiral arms is discarded in the comparison with the observations, and both groups assume a single pattern speed, which is probably not the case for real galaxies.

\section{Conclusions}

The position and strength of the shocks in barred galaxies derived from dynamical models is robust to the chosen model. Here, we compared a 2D to a 3D code, and despite the differences in the two codes and the small differences in the mass model, the resulting velocity and density maps for both models are very similar. However, for similar hydrodynamical parameters, the SPH code develops a central ring, associated to the $\mathrm{x}_{2}$ family of orbits while the gas distribution in the FS2 model does not present this central ring. This difference seems to be due to differences between the two codes and not to the difference in the treatment of the vertical forces. In any case, the position and the strength of the shocks in both models remains very similar. We can therefore conclude that the methodology used to derive the halo contribution to the potential using dynamical modelling of the gas flow in the bar region of spiral galaxies is robust to the codes used and to whether they are 2 or $3 \mathrm{D}$ codes.
Acknowledgements. I am very grateful to B. Weiner for providing all the necessary material that made this comparison possible. I am also grateful to Reynier Peletier and Almudena Zurita for the careful reading of the manuscript. I also acknowledge financial support from the Netherlands Organisation for Scientific Research (NWO) Foundation through a VENI grant. This work was also partly funded by the Plan Nacional del Espacio del Ministerio de Educación y Ciencia español. I also thank the referee for constructive comments that helped to improve this work.

\section{References}

Athanassoula, E. 1992, MNRAS, 259, 345

Benz, W. 1990, in The Numerical Modelling of Nonlinear Stellar Pulsations Problems and Prospects (Kluwer), NATO ASI Ser. C, 302, 269

Englmaier, P., \& Gerhard, O. 1997, MNRAS, 287, 57

Fux, R. 1997, Ph.D. Thesis, Geneva University

Fux, R. 1999, A\&A, 345, 787

Hockney, R. 1965, J. Assoc. Comput. Mach., 12, 95

Patsis, P., \& Athanassoula, E. 2000, MNRAS, 358, 45

Pérez, I. 2003, Ph.D. Thesis, Australian National University

Pérez, I., Fux, R., \& Freeman, K. 2004, A\&A, 424, 799

Pfenniger, D., \& Friedli, D. 1993, A\&A, 270, 561

Rautiainen, P., Salo, H., \& Buta, R. 2004, MNRAS, 349, 933

Salo, H., Rautiainen, P., Buta, R., et al. 1999, AJ, 117, 79

van Albada, G. 1985, A\&A, 142, 491

Weiner, B., Sellwood, J., \& Williams, T. 2001a, ApJ, 546, 931

Weiner, B., Williams, T., van Gorkom, J., \& Sellwood, J. 2001b, ApJ, 546, 916 